\title{
Energy-Efficient Parallel Two-Way Relaying Networks with Energy Harvesting
}

\author{
Nan $\mathrm{Qi}^{1}$, Huisheng Zhang ${ }^{1 *}$, Lin Zhang ${ }^{2}$, Lixin $\mathrm{Li}^{1}$, Ganning $\mathrm{He}^{1}$ \\ and Wenjing Wang ${ }^{1}$ \\ ${ }^{1}$ School of Electronics and Information, Northwestern Polytechnical University, \\ $X i$ 'an 710129, China \\ ${ }^{2}$ National Key Lab of Science and Technology on Communications, University of \\ Electronic Science and Technology of China, Chengdu 611731, China \\ qinan@mail.nwpu.edu.cn
}

\begin{abstract}
In this paper, the energy efficient parallel two-way relay (P-TWR) transmission networks with multiple energy harvesting nodes are studied. Relay selection (RS), power allocation (PA) and rate policies across multiple time intervals are jointly designed. The problem of energy efficiency (EE) maximization under the constraints of the energy causality and a pre-defined rate threshold is formulated. Though the optimization problem is NP-hard, we are able to convert it into a convex one. Specifically, by applying the Lagrange dual decomposition method, we separate PA from $R S$ and rate policy such that PA can be solved first. Further, with the obtained optimum PA solution, RS is mapped into a maximum weighted bipartite graph matching problem and solved with the Kuhn-Munkres algorithm. Finally, the optimum rate policy is obtained with the efficient interior point method. The performance comparisons with "Depleted Energy" and "Uniform Power" policies demonstrate that our policies can provide notable EE gains. It is also shown that a high pre-defined rate threshold costs a significant EE penalty. Finally, it is illustrated that the proposed algorithm converges speedily.
\end{abstract}

Keywords: parallel two-way relay; energy-efficiency; energy harvesting; Lagrange dual decomposition; maximum weighted bipartite graph matching

\section{Introduction}

In wireless sensor networks, the energy-deficient batteries may not be charged immediately due to physical environment constraints. Fortunately, energy harvesting (EH) enables the nodes to harvest energy from nature energy sources (e.g., such as solar, wind) and wirelessly refill their batteries [1]. However, the energy arrival is highly dependent on the specific environment such as the weather and location, which makes the harvested energy at an individual node intermittent available. As a consequence, energy management among the nodes comes to be a significant and challenging issue and has attracted considerable concerns recently [1]-[10]. Energy efficiency (EE), represented as the total number of successfully transmitted bits per joule energy, is a crucial metric to measure how efficiently the energy is consumed, especially for the EH networks. We study the energy efficient EH networks.

One effective technology to improve the EE is network coding (NC) [5]. Particularly, two-way relay (TWR) has been considered as a promising and easy-to-implement NC scheme, where one S-D pair exchanges messages via one relay [1]-[3]. Recent works focus on maximizing the throughput in energy harvesting TWR networks with infinite [1], [2] and finite battery storage [3]. Directional water-filling algorithm [4] was widely

* Corresponding Author 
applied to obtain the optimal power allocation (PA) policy that maximizes the throughput. For the TWR setups without EH, relay strategies including Decode-and-Forward (DF) [3], [6]-[7] and Amplify-and-Forward (AF) [8]-[10] and their effects on the EE were studied.

Although the aforementioned works have made significant contributions in the EH TWR techniques, they only studied the scenarios that a single S-D pair assisted by one/multiple fixed relays. In the practical scenario, however, it usually happens that several S-D pairs intend to exchange messages simultaneously via separate relays. We refer to it as parallel TWR (P-TWR) networks. In effect, the parallel setting model is classical and general since considerable communication channels can be transformed into parallel channels. For example, multiple-input multiple-output (MIMO) relay network [11] can be converted into parallel channels via matrix decompositions [12]. Also, TWR assisted orthogonal frequency division multiplexing (OFDM) can also be transformed into the P-TWR models due to the parallel nature of the sub-carriers [13], [14], [15]. However, to the best of our knowledge, EH P-TWR with consecutive transmissions was studied in few prior works. For the consecutive transmission scenario, as we will show later in the paper, the remaining energy from the prior transmissions affects the PA and relay selection (RS) in the later transmissions, thus energy management and RS in the current transmission cannot be "shortsighted" and have to take into account the system performance in the later transmissions. Hence, PA and RS have to be jointly optimized over multiple transmissions, i.e., the time dimension.

We are motivated to maximize the EE for EH P-TWR transmission networks. PA, RS and rate optimization will be jointly performed across consecutive transmissions according to the channel states and the amount of the harvested energy. Different from the aforementioned EH TWR networks in [1]-[4], our optimization dimension is increased with the existence of multiple S-D pairs competing for relays that have advantageous channel conditions. Moreover, the differences in channel conditions and harvested energy amount at the nodes make the P-TWR optimization problem challenging. We first formulate the EH P-TWR EE-maximization problem. With variable relaxation and fractional programming theory [14], we convert it into a convex form. Then a fast iteration algorithm is proposed based on the Lagrange dual decomposition. The closedform solutions for the optimal power at all nodes are obtained. After that, RS and rate policies are determined sequentially.

The rest of the paper is organized as follows. In Section 2, we present the system model. Problem formulation is given in Section 3. Then, we convert the optimization into a convex one in Section 4. The algorithm and optimal rate, power allocation and relay selection policies are given in Section 5. Numerical results are presented in Section 6. Section 7 concludes this paper.

\section{System Model}

In what follows, we will illustrate the data transmission and energy harvesting models, respectively.

\subsection{P-TWR Transmission}

We consider a network consisting of $M$ S-D pairs and $N(N \geq M)$ half-duplex relays, as depicted in Figure 1. All S-D pairs conduct communications with the assistance of their selected relays simultaneously. Specifically, every S-D pair is only connected with one relay; meanwhile, one relay at most assists one S-D pair at one time. The S-D pair and its relay constitute a transmission group. It is assumed that the channels for different S-R-D groups are independent (e.g., orthogonal in frequency) [12] and thus the potential interferences from the other S-R-D groups are avoided. For every group, we consider the two-phase protocol which consists of a multiple-access phase followed by a 
broadcast (MABC) phase [4]. In the following, let $S_{i}, D_{i}$ and $R_{j}$ represent the $i$ th source, its paired destination and the $j$ th relay, respectively.

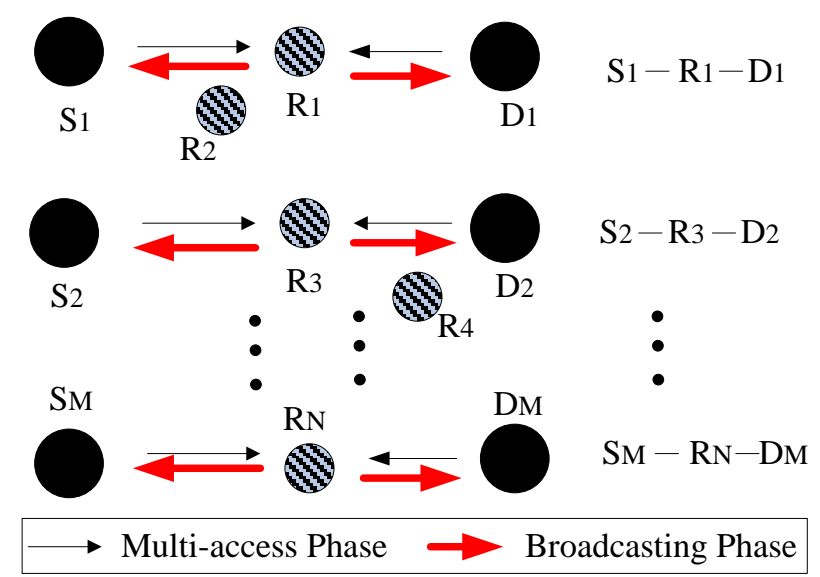

\section{Figure 1. An Example of P-TWR Transmission. $S_{1}-D_{1}, S_{2}-D_{2}$ and $S_{M}-D_{M}$ Pair Separately Selects $R_{1}, R_{3}$ and $R_{N}$}

In the first phase, all S-D pairs intend to conduct multiple access at the same time. Take $S_{i}-R_{j}-D_{i}$ group as an example. $S_{i}$ and $D_{i}$ simultaneously transmit their messages $X_{i, t}^{(s)}$ and $X_{i, t}^{(d)}$ to $R_{j}$ at time $t$. Denote the power of $S_{i}$ and $D_{i}$ as $p_{i, t}^{(s)}$ and $p_{i, t}^{(d)}$, respectively. We assume Rayleigh fading channels with zero-mean additive white Gaussian noise. Channel reciprocity is also assumed. At $R_{j}$, the received signals from $S_{i}$ and $D_{i}$ are respectively

$$
\begin{aligned}
& y_{i j, t}^{(s)}=h_{i j, t}^{(s)} \sqrt{p_{i, t}^{(s)}} X_{i, t}^{(s)}+n_{i j, t}^{(s)} \\
& y_{i j, t}^{(d)}=h_{i j, t}^{(d)} \sqrt{p_{i, t}^{(d)}} X_{i, t}^{(d)}+n_{i j, t}^{(d)}
\end{aligned}
$$

where $h_{i j, t}^{(s)}$ and $h_{i j, t}^{(d)}$ are respectively $S_{i}-R_{j}$ and $R_{j}-D_{i}$ channel gains. $n_{i j, t}^{(s)}$ and $n_{i j, t}^{(d)}$ are the noise items and their power is normalized to 1 .

In the second phase, all the selected relays in different S-R-D groups simultaneously decode their received messages and broadcast new generated messages to their separate S-D pairs. Take $S_{i}-R_{j}-D_{i}$ group as an example. $R_{j}$ first performs successive decoding and detects the messages from the $S_{i}-D_{i}$ pair. $y_{j, t}^{(r)}$ is generated at relays by XORing the decoded messages, i.e., $y_{j, t}^{(r)}=x_{i, t}^{(s)} \oplus x_{i, t}^{(d)}$. Note that “ $\oplus$ ” means the XOR operation. Then $R_{j}$ broadcasts $y_{j, t}^{(r)}$ to the $S_{i}-D_{i}$ pair with the power of $p_{j, t}^{(r)}$. Assume the channel state information (CSI) is known at the receivers. The S-D pair obtains their desired messages by removing the self-interference term. Take $x_{i, t}^{(d)}$ as an example. $x_{i, t}^{(d)}$ is obtained by $S_{i}$ according to the following operation:

$$
x_{i, t}^{(d)}=x_{i, t}^{(s)} \oplus y_{j, t}^{(r)}=x_{i, t}^{(s)} \oplus x_{i, t}^{(s)} \oplus x_{i, t}^{(d)}
$$




\subsection{Energy Harvesting Model}

We consider the off-line energy harvesting policy [4], in the sense that the harvested energy amount and harvesting time are known (or can be precisely predicted) to all nodes in advance. We claim an event occurs when either energy is harvested at any node or any channel in the network varies. As illustrated in Figure 2, we record all the event time into a time sequence and have $t_{1}=0<t_{2}<\cdots<t_{k}<\cdots<t_{K}$. The time period between $t_{k}$ and $t_{k+1}$ is defined as the $k$ th time interval with the duration of $\Gamma_{k}=t_{k+1}-t_{k}$. In total, $K$ time intervals are considered. Note that our time interval division model ensures that the channel gain in each individual time interval keeps constant as shown in Figure 2, which will facilitate the designs of PA, RS and rate policies.

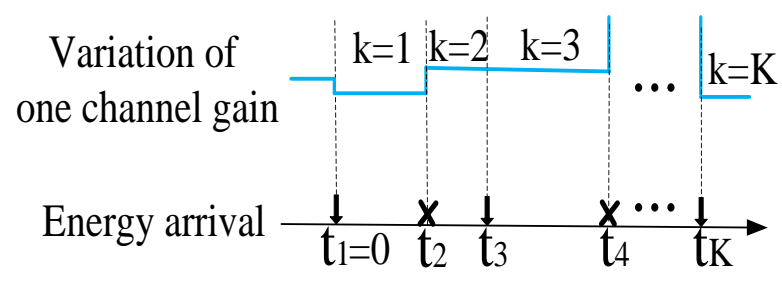

\section{Figure 2. An Illustration of the Combined Energy Harvesting and Channel Variation Events. The Bold Arrow Records the Moment When Energy Arrives While " $x$ " Means No Harvested Energy}

At the beginning of the $k$ th time interval (i.e., when $t=t_{k}$ ), we assume that $S_{i}$, $D_{i}$ and $R_{j}$ harvest $E S_{i, k}, E r_{j, k}$ and $E d_{i, k}$ joules of energy, respectively. The energy will be collected into an infinite battery [1], [2] before being consumed. Notice that the nodes do not necessarily harvest energy at the same time. That is, $E S_{i, k}, E r_{j, k}$ and $E d_{i, k}$ can be zero.

The fractions of the $k$ th interval allocated to the first phase and the second phase are denoted as $\Delta_{1, k}$ and $\Delta_{2, k}$, respectively. We have $\Delta_{1, k}+\Delta_{2, k}=1,0<\Delta_{1, k}, \Delta_{2, k}<1$. Then the consumed energies at $S_{i}, D_{i}$ and $R_{j}$ are respectively $p_{i, k}^{(s)} \Delta_{1, k} \Gamma_{k}, p_{i, k}^{(d)} \Delta_{1, k} \Gamma_{k}$ and $p_{j, k}^{(r)} \Delta_{2, k} \Gamma_{k}$ (Joules). Let $E s_{i, k}^{a v a}, E d_{i, k}^{\text {ava }}$ and $E r_{j, k}^{a v a}$ denote the available energy at the beginning of the $k$ th time interval, which include the accumulated energy during the $k-1$ intervals, i.e., prior to the $k$ th time interval and the newly harvested energy at $t_{k}$. In the following, we take $S_{i}$ as an example to derive the energy evolution equations. When $k=1$, the available energy at $S_{i}$ is

$$
E S_{i, 1}^{\text {ava }}=E S_{i, 1}+E S_{i, 0}, \forall i, k=1
$$

where $E S_{i, 0}$ denotes the initial storage energy, which is set as 0 .

For $k \geq 2$, the available energy at $S_{i}$ evolves as

$$
E S_{i, k+1}^{a v a}=E S_{i, k}^{\text {ava }}-p_{i, k}^{(s)} \Delta_{1, k} \Gamma_{k}+E S_{i, k+1}, k \geq 2
$$


where $\left(E S_{i, k}^{a v a}-p_{i, k}^{(s)} \Delta_{1, k} \Gamma_{k}\right)$ is the accumulated energy prior to the $(k+1)$ th time interval, and $E S_{i, k+1}$ is the newly harvested energy at $t_{k+1}$.

\section{Problem Formulation}

By definition, EE can be formulated as

$$
U_{E E}=\frac{L_{t o t}}{E_{t o t}}
$$

where $L_{\text {tot }}$ is the total number of successfully transmitted bits. Let $R_{i, k}^{(s)}$ and $R_{i, k}^{(d)}$ respectively denote the transmitting rate of $S_{i}$ and $D_{i}$ in the $k$ th time interval. Then

$$
L_{\text {tot }}=\sum_{k=1}^{K} \sum_{i=1}^{M}\left(R_{i, k}^{(s)}+R_{i, k}^{(d)}\right) \Gamma_{k}
$$

$E_{t o t}$ in (6) is the total consumed energy, which can be given as

$$
E_{t o t}=\sum_{k=1}^{K} \sum_{i=1}^{M} \sum_{j=1}^{N} u_{i j, k} \Gamma_{k}\left(p_{i, k}^{(s)} \Delta_{1, k}+p_{i, k}^{(d)} \Delta_{1, k}+p_{j, k}^{(r)} \Delta_{2, k}\right)
$$

Our objective is to maximize the objective function across $K$ intervals by jointly optimizing the power, rates and RS according to the CSI and the harvested energy amount.

The optimization problem is formulated as

$$
\begin{array}{ll}
\mathrm{P} 1: \max _{\mathrm{R}, \mathrm{P}, \mathrm{U}} U_{E E} \\
\text { s.t. } \mathrm{R} \in \mathrm{F}_{R}, \mathrm{P} \in \mathrm{F}_{E}, \mathrm{U} \in \mathrm{F}_{M}
\end{array}
$$

where $\mathrm{R}, \mathrm{P}$ and $\mathrm{U}$ are the set for all the rate, power and RS variables, respectively. $\mathrm{F}_{R}, \mathrm{~F}_{E}$ and $\mathrm{F}_{M}$ are the constraint set on rates, PA and RS. The constraint sets are formulated in what follows.

First, for RS, we introduce a binary variable $u_{i j, k}$ as below

$$
u_{i j, k}= \begin{cases}1 & \text { if } S_{i}-D_{i} \text { is matched with } R_{j} \\ 0 & \text { otherwise }\end{cases}
$$

The RS policy requires that every S-D pair be connected to only one relay, which is represented as $\sum_{j=1}^{N} u_{i j, k}=1$. Moreover, since one relay at most assists one S-D pair in any time interval, we have 0 羿 $\sum_{i=1}^{M} u_{i j, k} \quad 1$. These yield the constraint set $\mathrm{F}_{M}$ on RS, i.e.,

$$
\begin{aligned}
& \mathrm{F}_{M}=\left\{\left(u_{11, k}, u_{12, k}, \cdots, u_{M N, k}\right), \forall i, \forall j, \forall k:\right. \\
& \left.u_{i j, k}=\{0,1\}, \quad 0 \text { 羿 } \sum_{i=1}^{M} u_{i j, k} \quad 1, \quad \sum_{j=1}^{N} u_{i j, k}=1\right\}
\end{aligned}
$$

As for the energy constraints, since the energy not arriving yet cannot be consumed ahead of time due to the energy causality, it is required that the consumed energy cannot exceed the available amount. Correspondingly, the power constraint set $\mathrm{F}_{E}$ can be given as below 


$$
\begin{array}{r}
\mathrm{F}_{E}=\left\{\left(p_{i, k}^{(s)}, p_{j, k}^{(r)}, p_{i, k}^{(d)}\right), \forall i, \forall j, \forall k:\right. \\
0 \text { 翋 } p_{i, k}^{(s)} \Delta_{1, k} \Gamma_{k}=\sum_{j=1}^{N} u_{i j, k} p_{i, k}^{(s)} \Delta_{1, k} \Gamma_{k} \quad E S_{i, k}^{\text {ava }} \\
0 \text { 羿 } p_{i, k}^{(d)} \Delta_{1, k} \Gamma_{k}=\sum_{j=1}^{N} u_{i j, k} p_{i, k}^{(d)} \Delta_{1, k} \Gamma_{k} \quad E d_{i, k}^{\text {ava }} \\
\left.0 \text { 羿 } \sum_{i=1}^{M} u_{i j, k} p_{j, k}^{(r)} \Delta_{2, k} \Gamma_{k} \quad E r_{j, k}^{\text {ava }}\right\}
\end{array}
$$

We set the lower threshold on the transmission rate as $R_{i, k}^{(s)} \geq R_{i, \min }^{(s)}, R_{i, k}^{(d)} \geq R_{i, \min }^{(d)}$. Combining with the achievable rate region for TWR MABC system [4], we have the constraint set of rate and transmission power as below

$$
\begin{aligned}
& \mathrm{F}_{R}=\left\{\left(R_{1, k}^{(s)}, R_{2, k}^{(s)}, \cdots, R_{M, k}^{(s)}, R_{1, k}^{(d)}, R_{2, k}^{(d)}, \cdots, R_{M, k}^{(d)}\right), \forall i, \forall k:\right. \\
& R_{i, \text { min }}^{(s)} \text { 翋 } R_{i, k}^{(s)} \quad \sum_{j=1}^{N} u_{i j, k} \Delta_{1, k} \mathrm{C}\left(\mid h_{i j, k}^{(s)} \mathrm{P}^{2} p_{i, k}^{(s)} / \Delta_{1, k}\right) \\
& R_{i, \min }^{(s)} \text { 翋 } R_{i, k}^{(s)} \quad \sum_{j=1}^{N} u_{i j, k} \Delta_{2, k} \mathrm{C}\left(\mid h_{i j, k}^{(d)} \& p_{j, k}^{(r)} / \Delta_{2, k}\right) \\
& R_{i, \text { min }}^{(d)} \text { 羿 } R_{i, k}^{(d)} \quad \sum_{j=1}^{N} u_{i j, k} \Delta_{1, k} \mathrm{C}\left(\mid h_{i j, k}^{(d)} \& p_{j, k}^{(d)} / \Delta_{1, k}\right) \\
& R_{i, \min }^{(d)} \text { 羿 } R_{i, k}^{(d)} \quad \sum_{j=1}^{N} u_{i j, k} \Delta_{2, k} \mathrm{C}\left(\mid h_{i j, k}^{(s)} \& p_{j, k}^{(r)} / \Delta_{2, k}\right) \\
& \left.R_{i, k}^{(s)}+R_{i, k}^{(d)} 》 \sum_{j=1}^{N} u_{i j, k} \Delta_{1, k} \mathrm{C}\left(\frac{\left|h_{i j, k}^{(s)} \mathcal{R} p_{i, k}^{(s)}+\right| h_{i j, k}^{(d)} \mathcal{R} p_{i, k}^{(d)}}{\Delta_{1, k}}\right)\right\}
\end{aligned}
$$

where $\mathrm{C}(p)=\log (1+p)$.

Due to the existence of binary variable $u_{i j, k}, \mathrm{P} 1$ is a mixed integer programming problem. Moreover, the non-concave/convex fractional structure of the objective function makes P1 non-concave/convex. To obtain the solution with a reduced complexity, we reformulate $\mathrm{P} 1$ and convert it into a convex problem.

\section{Problem Reformulation}

The Dinkelbach's method [14] is first applied to transform the objective function into its subtractive form. Then by relaxing $u_{i j, k}$ into a continuous variable, we formulate a dual problem.

\subsection{Nonlinear Fractional Problem Transformation}

We have the following Lemma for P1 [14].

Lemma 1. The resource allocation policy can achieve the maximum energy efficiency

if and only if

$$
q^{*}=\max U_{E E}
$$

$$
V\left(q^{*}\right)=V\left(q^{*}, \mathrm{P}^{*}, \mathrm{R}^{*}, \mathrm{U}^{*}\right)=\max \left(L_{t o t}-q E_{t o t}\right)=0
$$


In Lemma 1, $q^{*}$ is the maximum EE to be determined.

According to Lemma 1, we reformulate P1 as P2.

$$
\mathrm{P} 2: \max _{\mathrm{R}, \mathrm{P}, \mathrm{U}} V(q)=L_{t o t}-q E_{t o t}
$$

In Dinkelbach's method, $q$ is iteratively updated. In every iteration, with a given $q$, we only need to focus on searching $\mathrm{R}^{*}, \mathrm{P}^{*}$ and $\mathrm{U}^{*}$ that makes $V(q)$ converge to a given tolerance. If not, $q$ is updated and we repeat the maximization problem until $V(q)$ converges or the iteration reaches the maximal iterations.

In the following subsection, we further reformulate P2 and convert it into a convex problem such that $\mathrm{R}^{*}, \mathrm{P}^{*}$ and $\mathrm{U}^{*}$ can be found efficiently.

\subsection{Dual Problem Reformulation}

We relax $u_{i j, k}$ into a continuous variable, i.e., $0 \leq u_{i j, k} \leq 1 . u_{i j, k}$ can be interpreted as a time-sharing factor in assigning the $j$ th relay to the $i$ th S-D pair in the $k$ th interval. In addition, we introduce three new variables which represent the transmission power on $S_{i}-R_{j}, R_{j}-D_{i}$ and $S_{i}-R_{j}$ (or $D_{i}-R_{j}$ ) links under the timesharing condition, i.e.,

$$
\tilde{p}_{i j, k}^{(r)}=u_{i j, k} p_{i j, k}^{(r)}, \tilde{p}_{i j, k}^{(s)}=u_{i j, k} p_{i j, k}^{(s)}, \tilde{p}_{i j, k}^{(d)}=u_{i j, k} p_{i j, k}^{(d)}
$$

By substituting (23) into P2, we obtain

$$
\mathrm{P} 3: \max V=L_{t o t}-q E_{t o t}(\tilde{\mathrm{P}} ; \mathrm{U}, \mathrm{R})
$$

$$
\text { s.t. } \mathrm{R} \in \mathrm{F}_{R}, \tilde{\mathrm{P}} \in \mathrm{F}_{E}, 0 \leq u_{i j, k} \leq 1, \forall i, j, k
$$

where $\tilde{\mathrm{P}} ;=\left\{\tilde{P}_{i j, k}^{s}, \tilde{p}_{i j, k}^{(d)}, \tilde{p}_{i j, k}^{(r)}, \forall i, \forall j, \forall k\right\}$. It can be verified that P3 is jointly convex with respect to $\tilde{P}$; $U$ and $R$. For proof, see Appendix A.

The Lagrange dual function of P3 is $\mathrm{L}(\mathrm{R}, \tilde{\mathrm{P}}, \mathrm{U}, \boldsymbol{\lambda}, \boldsymbol{\delta}, \boldsymbol{\theta})$

$$
\begin{aligned}
= & L_{t o t}-q E_{t o t}-\sum_{i=1}^{M} \sum_{k=1}^{K} \lambda_{i, k}^{(s)}\left(\sum_{j=1}^{N} \tilde{p}_{i j, k}^{(s)}-\frac{E s_{i, k}^{\text {vva }}}{\Delta_{1, k} \Gamma_{k}}\right)-\sum_{i=1}^{M} \sum_{k=1}^{K} \lambda_{i, k}^{(d)}\left(\sum_{j=1}^{N} \tilde{p}_{i j, k}^{(d)}-\frac{E d_{i, k}^{a v a}}{\Delta_{1, k} \Gamma_{k}}\right) \\
& -\bar{\delta}_{i, k}^{(s)}\left(R_{i, \min }^{(s)}-R_{i, k}^{(s)}\right)-\sum_{j=1}^{N} \sum_{k=1}^{K} \lambda_{j, k}^{(r)}\left(\sum_{i=1}^{M} \tilde{p}_{i j, k}^{(r)}-\frac{E r_{j, k}^{a v a}}{\Delta_{2, k} \Gamma_{k}}\right)-\bar{\delta}_{i, k}^{(d)}\left(R_{i, \min }^{(d)}-R_{i, k}^{(d)}\right) \\
& -\sum_{i=1}^{M} \sum_{k=1}^{K} \delta_{i, k}^{(s)}\left(R_{i, k}^{(s)}-\sum_{j=1}^{N} u_{i j, k} \Delta_{1, k} \mathrm{C}\left(\frac{\left|h_{i j, k}^{(s)}\right|^{2} \tilde{p}_{i j, k}^{(s)}}{\Delta_{1, k} u_{i j, k}}\right)-\sum_{i=1}^{M} \sum_{k=1}^{K} \hat{\delta}_{i, k}^{(s)}\left(R_{i, k}^{(s)}-\sum_{j=1}^{N} u_{i j, k} \Delta_{2, k} \mathrm{C}\left(\frac{\left|h_{i j, k}^{(d)}\right|^{2} \tilde{p}_{i j, k}^{(r)}}{\Delta_{2, k} u_{i j, k}}\right)\right)\right. \\
& -\sum_{i=1}^{M} \sum_{k=1}^{K} \delta_{i, k}^{(d)}\left(R_{i, k}^{(d)}-\sum_{j=1}^{N} u_{i j, k} \Delta_{1, k} \mathrm{C}\left(\frac{\left|h_{i j, k}^{(d)}\right|^{2} \tilde{p}_{i j, k}^{(d)}}{\Delta_{1, k} u_{i j, k}}\right)-\sum_{i=1}^{M} \sum_{k=1}^{K} \hat{\delta}_{i, k}^{(d)}\left(R_{i, k}^{(d)}-\sum_{j=1}^{N} u_{i j, k} \Delta_{2, k} \mathrm{C}\left(\frac{\left.h_{i j, k}^{(s)}\right|^{2} \tilde{p}_{i j, k}^{(r)}}{\Delta_{2, k} u_{i j, k}}\right)\right)\right. \\
& -\sum_{i=1}^{M} \sum_{k=1}^{K} \delta_{i, k}\left(R_{i, k}^{(s)}+R_{j, k}^{(d)}-\sum_{j=1}^{N} u_{i j, k} \Delta_{1, k} \mathrm{C}\left(\frac{\left|h_{i j, k}^{(s)}\right|^{2} \tilde{p}_{i j, k}^{(s)}+\left|h_{i j, k}^{(d)}\right|^{2} \tilde{p}_{i j, k}^{(d)}}{\Delta_{1, k} u_{i j, k}}\right)\right. \\
& -\sum_{j=1}^{N} \sum_{k=1}^{K} \theta_{j, k}^{(r)}\left(\sum_{i=1}^{M} u_{i j, k}-1\right)-\sum_{i=1}^{M} \sum_{k=1}^{K} \theta_{j, k}^{(s)}\left(\sum_{j=1}^{N} u_{i j, k}-1\right)
\end{aligned}
$$


where $\boldsymbol{\lambda}=\left\{\left(I_{i, k}^{(s)}, l_{i, k}^{(d)}, I_{j, k}^{(r)}\right), " i, j, k\right\}, \quad \boldsymbol{\delta}=\left\{\left(d_{i, k}^{(s)}, d_{i, k}^{(d)}, \hat{d}_{i, k}^{(s)}, \hat{y}_{i, k}^{(d)}, \bar{d}_{i, k}^{(s)}, \bar{d}_{i, k}^{(d)}, d_{i, k}\right), \forall i, k\right\}$, $\boldsymbol{\theta}=\left\{\left(q_{j, k}^{(r)}, q_{j, k}^{(s)}\right), " j, k\right\}$ are the Lagrange multipliers for energy causality, rate constraints and RS, respectively. All the multipliers are all nonnegative.

Finally, the dual problem can be formulated as

$$
\mathrm{P} 4: \min _{\lambda, \delta, \theta \cdot 0} \max _{\mathrm{R}, \tilde{\mathrm{P}}, U} \mathrm{~L}(\mathrm{R}, \tilde{\mathrm{P}}, \mathrm{U}, \lambda, \delta, \theta)
$$

Since P3 is convex, the duality gap between P3 and P4 is zero. That is, P3 can be equivalently solved if the optimum solution for P4 is determined. The solution of P4 can be obtained by Lagrange dual decomposition. Specifically, we separate P4 into two levels. The lower level aims at solving the inner maximization, i.e., $\max _{\mathrm{R}, \mathrm{P}, \mathrm{U}} \mathrm{L}(\mathrm{R}, \tilde{\mathrm{P}}, \mathrm{U}, \boldsymbol{\lambda}, \boldsymbol{\delta}, \theta)$ with fixed $\boldsymbol{\lambda}, \boldsymbol{\delta}$ and $\boldsymbol{\theta}$. The higher level focuses on solving the outer minimization, i.e., $\min _{\lambda, \delta, \theta \cdot 0} \mathrm{~L}(\mathrm{R}, \tilde{\mathrm{P}}, \mathrm{U}, \lambda, \delta, \theta)$ with obtained $\mathrm{R}, \mathrm{P}$ and U. The two levels proceed iteratively. Outer minimization can be easily solved with the efficient and well-known gradient method [16]. In this paper, we put emphasis on the inner maximization, which is depicted in Section 5.

\section{Optimal PA, RS and Rate Policies}

\subsection{Suboptimal Power Allocation}

For large $x$, we have $\log (1+X) \approx \log (X)$. Then in the high SNR region, we have $\mathrm{C}\left(\left|h_{i j, k}^{(\Lambda)}\right|^{2} \tilde{p}_{i j, k}^{(\Lambda)} / \Delta_{1, k} u_{i j, k}\right) \approx \log \left(\left|h_{i j, k}^{(\Lambda)}\right|^{2} \tilde{p}_{i j, k}^{(\Lambda)} / \Delta_{1, k} u_{i j, k}\right)$, where $\Lambda \in\{s, d\}$. Since P3 is convex, strong duality holds and KKT conditions can be employed to solve the inner maximization in P4. The KKT conditions specify that the partial derivatives of (25) with respect to $\tilde{p}_{i j, k}^{(s)}$ and $\tilde{p}_{i j, k}^{(d)}$ should be zero, we have

$$
\begin{aligned}
& \frac{\partial \mathrm{L}}{\partial \tilde{p}_{i j, k}^{(s)}}=-Z_{i, k}^{(s)}+\frac{d_{i, k}^{(s)} u_{i j, k} \mathrm{D}_{1, k}}{\tilde{p}_{i j, k}^{(s)}}+\frac{d_{i, k} \mid h_{i j, k}^{(s)} P^{2} u_{i j, k} \mathrm{D}_{1, k}}{\left|h_{i j, k}^{(s)}\right|^{2} \tilde{p}_{i j, k}^{(s)}+\left|h_{i j, k}^{(d)}\right|^{2} \tilde{p}_{i j, k}^{(d)}}=0
\end{aligned}
$$

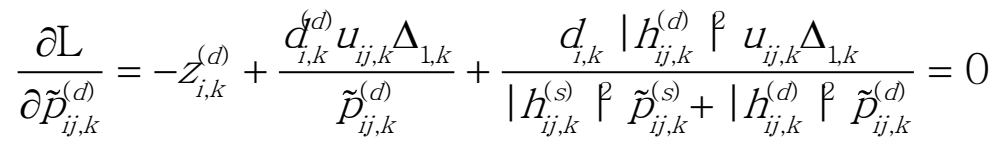

In (27) and (28), $Z_{i, k}^{(s)}$ and $Z_{i, k}^{(d)}$ are

$$
\begin{aligned}
& Z_{i, k}^{(s)}=\left(q \Delta_{1, k} \Gamma_{k}+\lambda_{i, k}^{(s)}+\sum_{l=k+1}^{K} \lambda_{i, l}^{(s)} \frac{\Delta_{1, k} \Gamma_{k}}{\Delta_{1, I} \Gamma_{i, l}}\right) \ln (2)>0 \\
& Z_{i, k}^{(d)}=\left(q \Delta_{1, k} \Gamma_{k}+\lambda_{i, k}^{(d)}+\sum_{l=k+1}^{K} \lambda_{i, l}^{(d)} \frac{\Delta_{1, k} \Gamma_{k}}{\Delta_{1, I} \Gamma_{i, l}}\right) \ln (2)>0
\end{aligned}
$$

Note that $Z_{i, k}^{(s)}$ and $Z_{i, k}^{(d)}$ are positive, which is due to the fact that $q \Delta_{1, k} \Gamma_{k}>0$ and all the Lagrange multipliers are all nonnegative.

Let $p_{i, k}^{\left(S^{*}\right)}, \quad p_{i, k}^{\left(d^{*}\right)}$ and $p_{j, k}^{\left(r^{*}\right)}$ respectively denote the optimum power at $S_{i}, D_{i}$ and $R_{j}$. We have the following proposition.

Proposition 1. In the high SNR region, there is only one optimal nonnegative solution respectively for $p_{i, k}^{\left(S^{*}\right)}, \quad p_{i, k}^{\left(d^{*}\right)}$ and $p_{j, k}^{\left(r^{*}\right)}$. Specifically, 


$$
p_{i, k}^{\left(d^{*}\right)}= \begin{cases}\frac{-f_{2}+\sqrt{f_{2}^{2}-4 f_{3} f_{1}}}{2 f_{3}}, & f_{3}>0 \\ \frac{E d_{i, k}^{\text {ava }}}{\Delta_{1, k} \Gamma_{i, k}}, & f_{3}<0, f_{2}^{2}-4 f_{3} f_{1}<0 \\ \frac{-f_{2}}{2 f_{3}}, & f_{3}<0, f_{2}^{2}-4 f_{3} f_{1}=0 \\ \frac{-f_{1}}{f_{2}}, & f_{3}=0\end{cases}
$$

where

$$
\begin{aligned}
& f_{3}=\mid h_{i j, k}^{(d)} \& Z_{i, k}^{(d)} b_{i j, k}^{(s)} \\
& f_{2}=\left|h_{i j, k}^{(d)} \& Z_{i, k}^{(d)} c_{i j, k}^{(s)}+\right| h_{i j, k}^{(d)} \& Z_{i, k}^{(s)} a_{i j, k}^{(s)}-d_{i, k}^{(d)} \mathrm{D}_{1, k}\left|h_{i j, k}^{(d)} \& b_{i j, k}^{(s)}-d_{i, k} \mathrm{D}_{1, k}\right| h_{i j, k}^{(d)} \& b_{i j, k}^{(s)} \\
& f_{1}=-c_{i j, k}^{(s)} \mathrm{D}_{1, k} \mid h_{i j, k}^{(d)} P\left(d_{i, k}^{(s)}+d_{i, k}^{(d)}+d_{i, k}\right) \\
& a_{i j, k}^{(s)}=d_{i, k}^{(s)} \mathrm{D}_{1, k}\left|h_{i j, k}^{(d)} \& ? 0 ; c_{i j, k}^{(s)}=d_{i, k}^{(d)} \mathrm{D}_{1, k}\right| h_{i j, k}^{(s)} P^{2} \text { ? } 0 \\
& b_{i j, k}^{(s)}=\left|h_{i j, k}^{(d)} \& z_{i, k}^{(s)}-\right| h_{i j, k}^{(s)} \& z_{i, k}^{(d)}
\end{aligned}
$$

$p_{i, k}^{\left(S^{*}\right)}$ is paired with $p_{i, k}^{\left(d^{*}\right)}$ and can be directly obtained by exchanging " $d$ " in (31) with " $s " . p_{j, k}^{\left(r^{*}\right)}$ can be obtained by solving $\frac{\partial \mathrm{L}}{\partial \tilde{p}_{i j, k}^{(r)}}=0$. We ignore their explicit closedform expressions here.

Proof. The proof can be found in Appendix B.

Remark 1. We observe that $p_{i, k}^{\left(d^{*}\right)}$ is related with $Z_{i, k}^{(s)}$ and $Z_{i, k}^{(d)}$. Interestingly, as shown in (29) and (30), $Z_{i, k}^{(s)}$ and $Z_{i, k}^{(d)}$ are not only associated with the Lagrange multipliers of the current time interval, i.e., $\lambda_{i, k}^{(s)}, \lambda_{i, k}^{(d)}$ but also with those of $(k+1)$ th, $(k+2)$ th, $\cdots, K$ th intervals. Similar conclusions can be obtained for $p_{j, k}^{\left(r^{* *}\right)}$. It can be further noticed that the Lagrange multipliers are corresponding to the energy causality constraints and affected by the transmitting power. In this sense, PA policies in different time intervals are interrelated via the multipliers.

Remark 2. The transmitting power decreases with the channel gains. That is, $\frac{\partial p_{i, k}^{\left(s^{*}\right)}}{\partial\left|h_{i j, k}^{(s)}\right|^{2}}<0, \frac{\partial p_{i, k}^{\left(d^{*}\right)}}{\partial\left|h_{i j, k}^{(d)}\right|^{2}}<0, \frac{\partial p_{j, k}^{\left(r^{*}\right)}}{\partial\left|h_{i j, k}^{(s)}\right|^{2}}<0$ and $\frac{\partial p_{j, k}^{\left(r^{*}\right)}}{\partial\left|h_{i j, k}^{(d)}\right|^{2}}<0$.

It is intuitively reasonable, since less power is needed to transmit messages on the channels of larger channel gains. For strict proof, see Appendix C.

\subsection{Suboptimal Relay Selection}

We introduce a parameter, $W_{i j, k}$, given below: 


$$
\begin{aligned}
& W_{i j, k}=\left.\frac{\mathbb{} \mathrm{L}}{\mathbb{\Upsilon} u_{i j, k}}\right|_{\mathrm{P}=\mathrm{P}^{*}}
\end{aligned}
$$

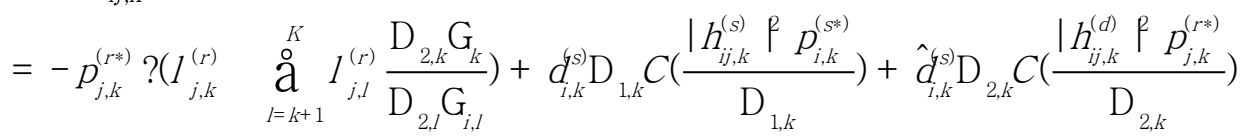

$$
\begin{aligned}
& -q_{j, k}^{(r)}-q_{j, k}^{(s)}+d_{i, k}^{(d)} \mathrm{D}_{1, k} C\left(\frac{\mid h_{i j, k}^{(d)} f p_{i, k}^{(d *)}}{\mathrm{D}_{1, k}}\right)+\hat{d}_{i, k}^{(d)} \mathrm{D}_{2, k} C\left(\frac{\mid h_{i j, k}^{(s)} f^{2} p_{j, k}^{(r *)}}{\mathrm{D}_{2, k}}\right) \\
& +d_{i, k} \mathrm{D}_{1, k} C\left(\frac{\left|h_{i j, k}^{(s)} \mathcal{R} p_{i, k}^{\left(s^{*}\right)}+\right| h_{i, k}^{(d)} \mathcal{R} p_{i, k}^{(d *)}}{\mathrm{D}_{1, k}}\right)
\end{aligned}
$$

which is called the marginal benefit provided to the system by assigning $R_{j}$ to the $S_{i}-D_{i}$ pair in the $k$ th time interval. Interestingly, $W_{i j, k}$ is related with $\mathrm{P}^{*}$ and independent from the rate variables, which facilitates RS though the rate policy has not been determined yet.

Remark 3. Having revealed that PA policies in different time intervals mutually affect and that RS depends on PA, we obtain the insight that RS in different time intervals also interacts via the Lagrange multipliers.

Then optimizing RS for all the S-D pairs is equivalent to maximizing the sum marginal benefits, which is formulated in P5.

$$
\text { P5: } \max \sum_{k} \sum_{j} \sum_{i} u_{i j, k} w_{i j, k}
$$

s.t. (7)

For P5, we have the following proposition.

Proposition 2. Given $\mathrm{P}^{*}$, P5 can be transformed into a maximum weighted bipartite graph matching (MWBGM) problem.

Proof. See Appendix D.

\subsection{Suboptimal Rate Policy}

Once the optimum PA and RS policies are determined, P4 is reduced into a standard convex rate optimization problem. Suboptimal rates can be obtained with the classical interior point method [16].

\section{Numerical Results}

Assume that there are two S-D pairs and 4 relays. 10 time intervals with a time span of 4.534 seconds are considered. The durations of each individual time interval are nonuniform, which will be shown in the horizontal axis of the following figures. Additionally, we assume that $R_{i, \min }^{(s)}=R_{i, \min }^{(d)}=R_{\min }$. The below results are obtained on Matlab.

\subsection{Performance Analyses}

In Figure 3, the exhaustive search and suboptimal results are first obtained for the scenarios when $R \min =9,9.5,10$ (bps). It is shown that the suboptimal results closely match with the exhaustive search results, which verifies our algorithm.

Additionally, we depict the EE obtained by another two transmission strategies. In the "Depleted Energy" scheme, the harvested energy is used up within every time interval. In the "Uniform Power" policy, all the nodes transmit with uniform power obtained by averaging all the harvested energy in 10 time intervals among all the nodes. In the above 
two strategies, RS is optimized with the Kuhn-Munkres algorithm. As can be seen clearly, our suboptimal policy outperforms the other two strategies.

Moreover, as can be seen, the increase in the rate threshold comes at the expense of a reduction in the EE. Specifically, compared with the scenarios where $R_{\min }=9$, the EE respectively falls by $47 \%$ and $74 \%$ when $R \min$ increases to 9.5 and 10 bps. This is due to the fact that to achieve higher rate threshold, more energy is enforced to be consumed on the channels of lower gains, thus decreasing the overall EE.

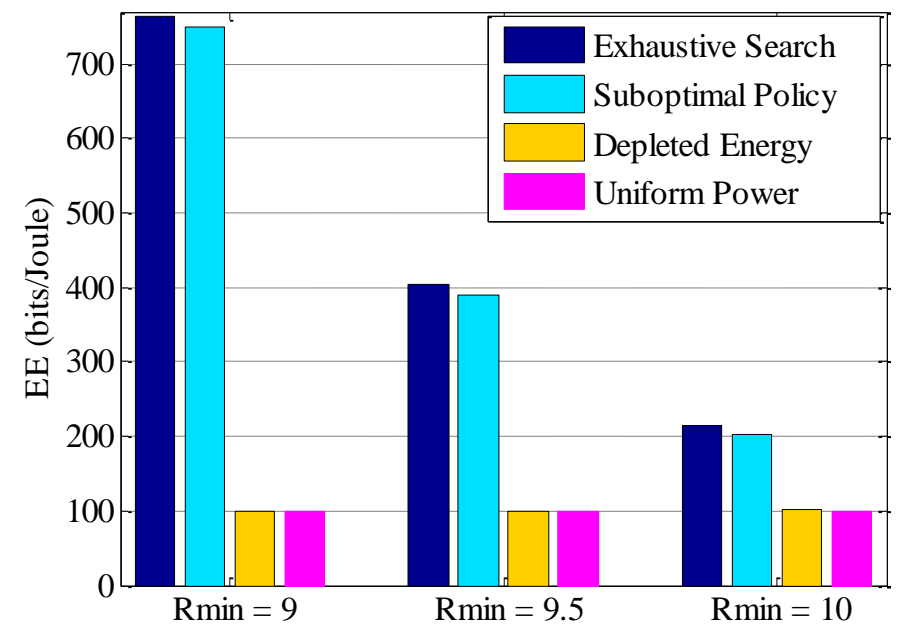

Figure 3. EE Comparison among Different Transmission Strategies

\subsection{Optimal Policy Illustration}

The cumulative harvested energy and the optimal PA policy are depicted in Figure 4 $\sim$ Figure 7 . We set $R \min$ as $10 \mathrm{bps}$. For the cumulative harvested energy curves, the rising height at the beginning of every time interval represents the newly harvested energy amount. Zero-rising height implies that no energy is harvested. Meanwhile, for the optimal PA curve, the ordinate values represent the amount of total consumed energy at one specific node. Correspondingly, the slope of one line segment represents the data transmission power in the corresponding time interval.

From Figure 4 Figure 7, we observe that the optimal power policy curves are not higher than the available energy curves due to the energy causality constraint. Moreover, in some transmission periods, the optimal consumed energy amount is not necessarily equal to that of the cumulative harvested. In other words, some energy in the prior time intervals is saved and consumed in the later intervals for the sake of maximizing the energy efficiency. Take $D_{2}$ in Figure 5 as an example, the available energies are not used up till the 8th time interval. 


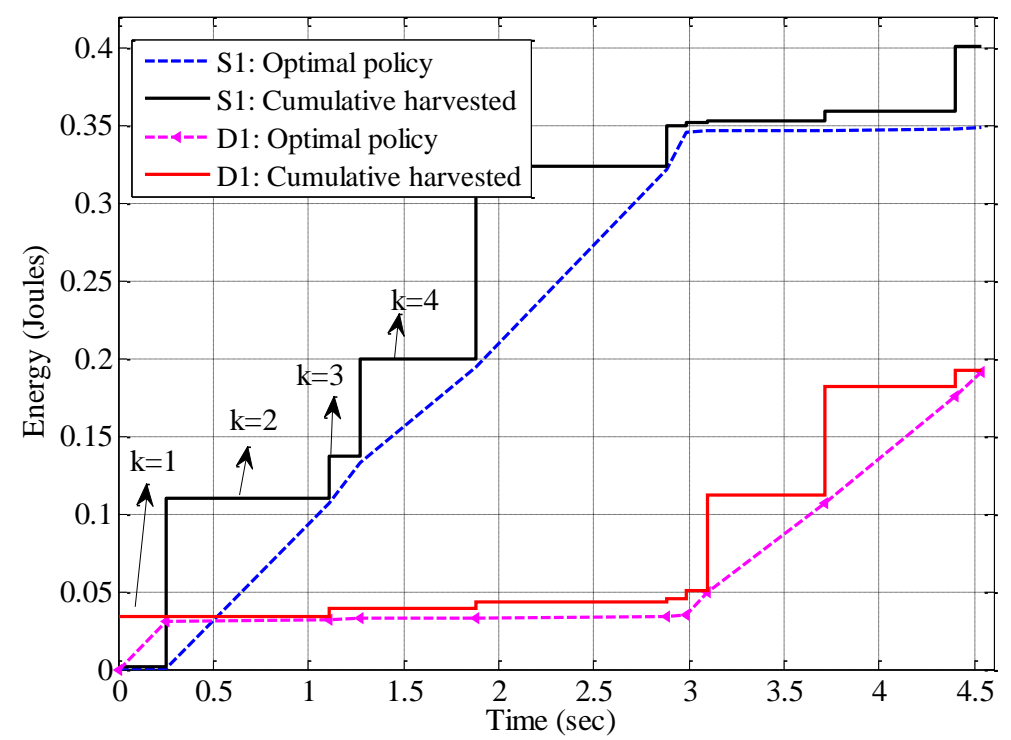

Figure 4. Optimal policy for $S_{1}-D_{1}$ pair

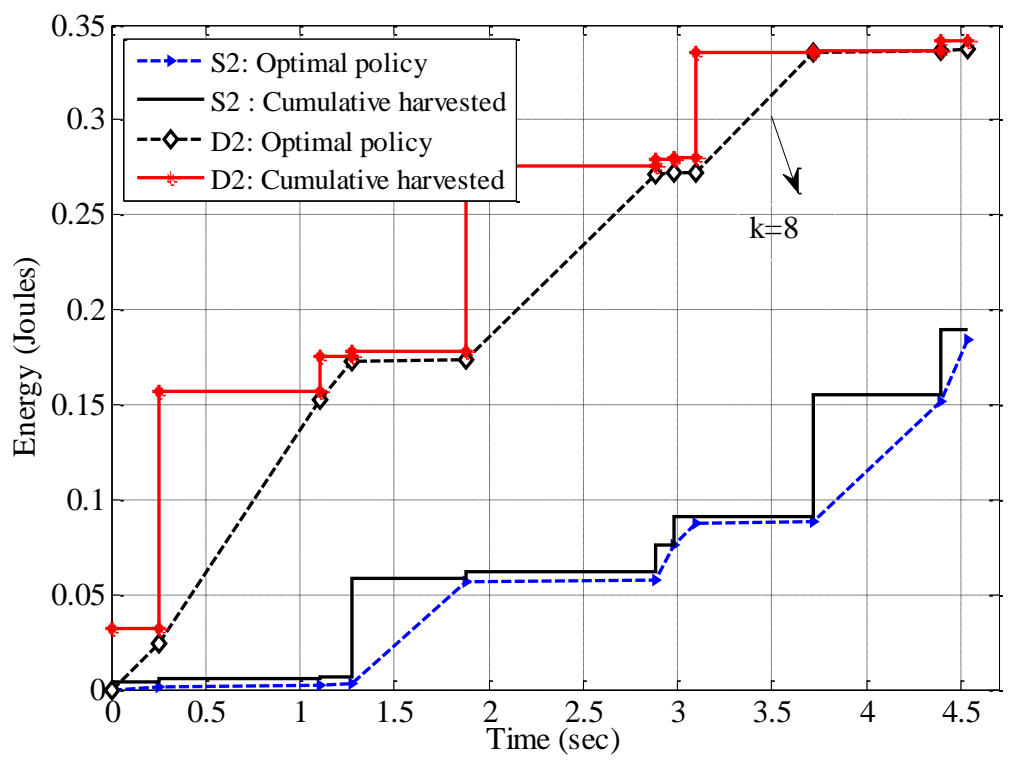

Figure 5. Optimal policy for $S_{2}-D_{2}$ pair

In Figure 6 and Figure 7, a zero-slope of an optimal policy curve indicates that one relay is not selected in the corresponding time interval. Note that for any time interval, only two out of four line segments are zero-slope, which means only two relays are selected in each individual time interval. The detailed RS results are shown in Table 1. It can be found that our RS policy provides S-R-D groups with larger channel gains. Take RS in the 2nd and 3rd time intervals as examples. Our algorithm results show that $S_{l}-D_{1}$ pair keeps selecting $R_{1}$, while $S_{2}-D_{2}$ selects $R_{2}$ in the 2 nd time interval and $R_{4}$ in the 3 rd time interval. Coherently, we see from Figure 8 that $S_{1}-D_{1}$ and $R_{1}-D_{1}$ channel gains in both time intervals are the largest compared with the other possible RS choices for the $S_{1}-D_{1}$ pair. Meanwhile, the inter-channels of the $S_{2}-R_{2}-D_{2}$ group in the 2nd time interval and the $S_{2}-R_{4}-D_{2}$ group in the 3rd time interval also have larger gains. 


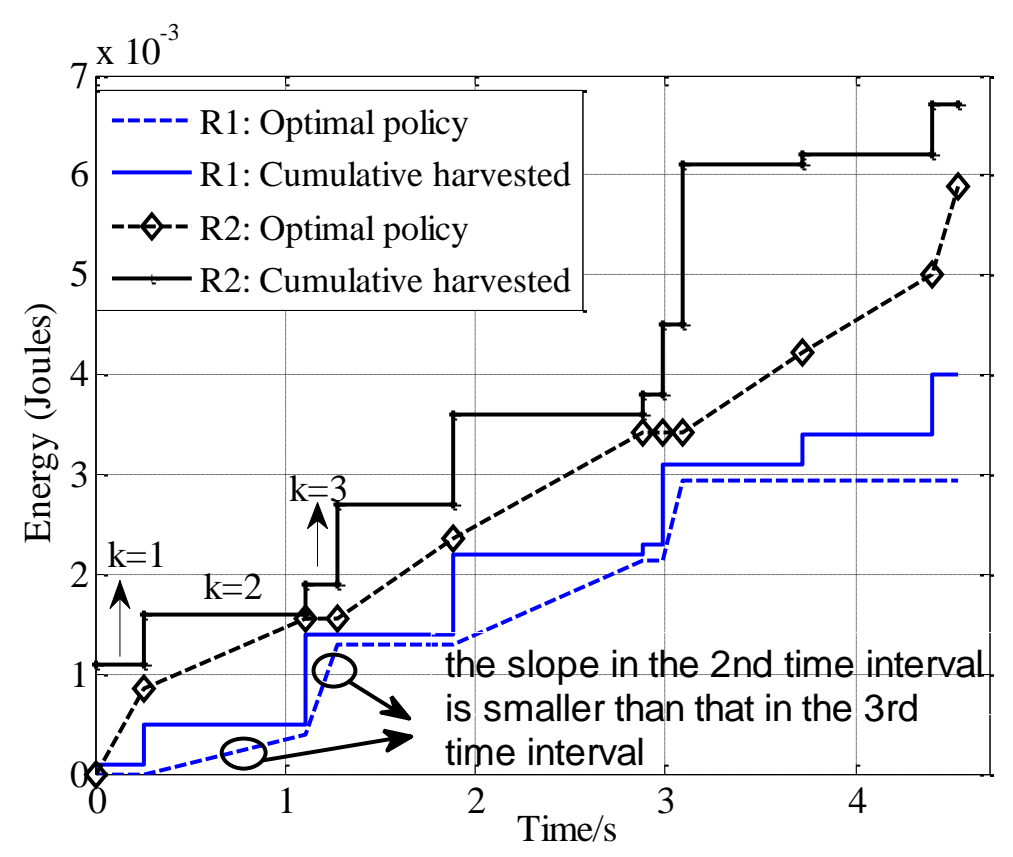

Figure 6. Optimal policy for $\mathbf{R}_{1}, \mathbf{R}_{2}$

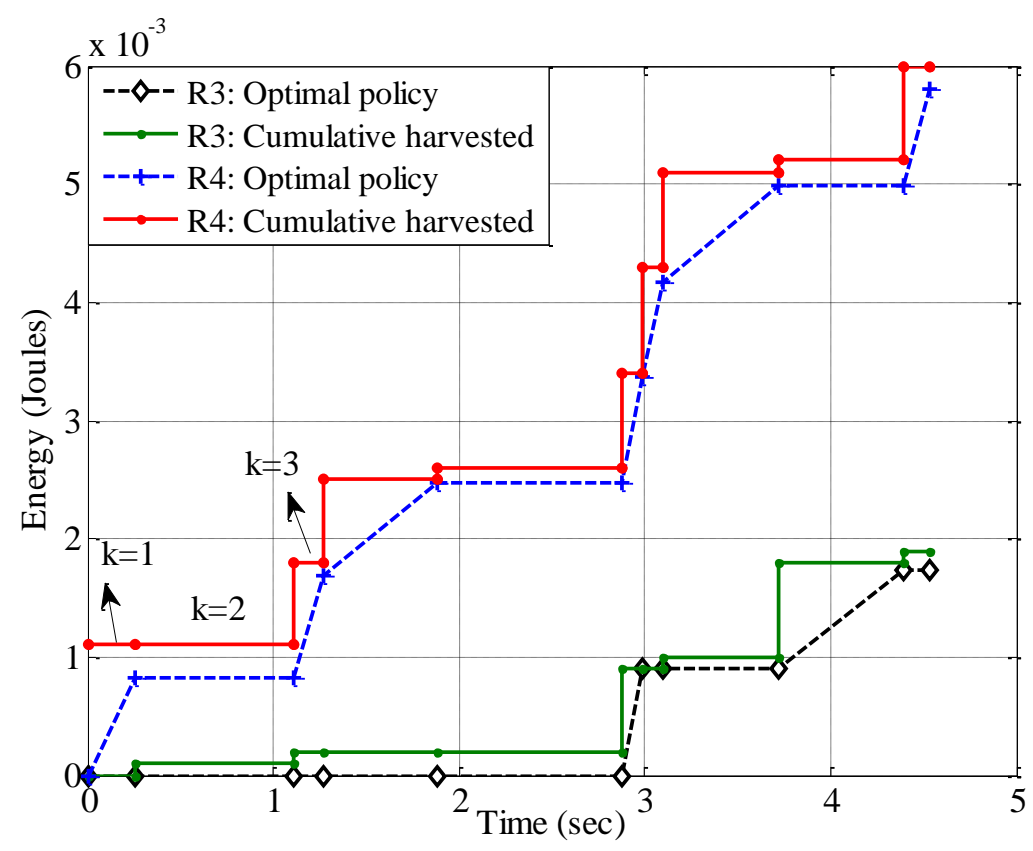

Figure 7. Optimal Policy for $\mathbf{R}_{\mathbf{3}}, \mathbf{R}_{\mathbf{4}}$ 
Table 1. RS Results

\begin{tabular}{|c|c|c|c|c|c|c|c|c|c|c|}
\hline Relays $\Gamma$ & $\Gamma_{1}$ & $\Gamma_{2}$ & $\Gamma_{3}$ & $\Gamma_{4}$ & $\Gamma_{5}$ & $\Gamma_{6}$ & $\Gamma_{7}$ & $\Gamma_{8}$ & $\Gamma_{9}$ & $\Gamma_{10}$ \\
\hline $\mathrm{R}_{1}$ & & $\dagger$ & $\dagger$ & & $\dagger$ & & $\dagger \dagger$ & & & \\
\hline $\mathrm{R}_{2}$ & $+\dagger$ & $\dagger \dagger$ & & $\dagger$ & $\dagger \dagger$ & & & $\dagger \dagger$ & $\dagger \dagger$ & $\dagger \dagger$ \\
\hline $\mathrm{R}_{3}$ & & & & & & $\dagger \dagger$ & & & $\dagger$ & \\
\hline $\mathrm{R}_{4}$ & $\dagger$ & & $\dagger \dagger$ & $\dagger \dagger$ & & $\dagger$ & $\dagger$ & $\dagger$ & & $\dagger$ \\
\hline
\end{tabular}

Moreover, for the optimal policy curve of $R_{I}$ shown in Figure 6, the slope of the line segment in the 2 nd time interval is smaller than that in the 3 rd time interval. It indicates that the transmitting power of $R_{l}$ in the 2 nd time interval is smaller than that in the 3 rd time interval. This is reasonable, since $S_{l}-D_{l}$ and $R_{l}-D_{l}$ channel gains (i.e., $\left|h_{11}^{(s)}\right|$ and $\left|h_{11}^{(d)}\right|$ ) in the 2rd time interval are both larger than those in the 3rd time interval as shown in Figure 8, and less power is needed. It is also in accordance with Remark 2 and hereby verifies our conclusion.
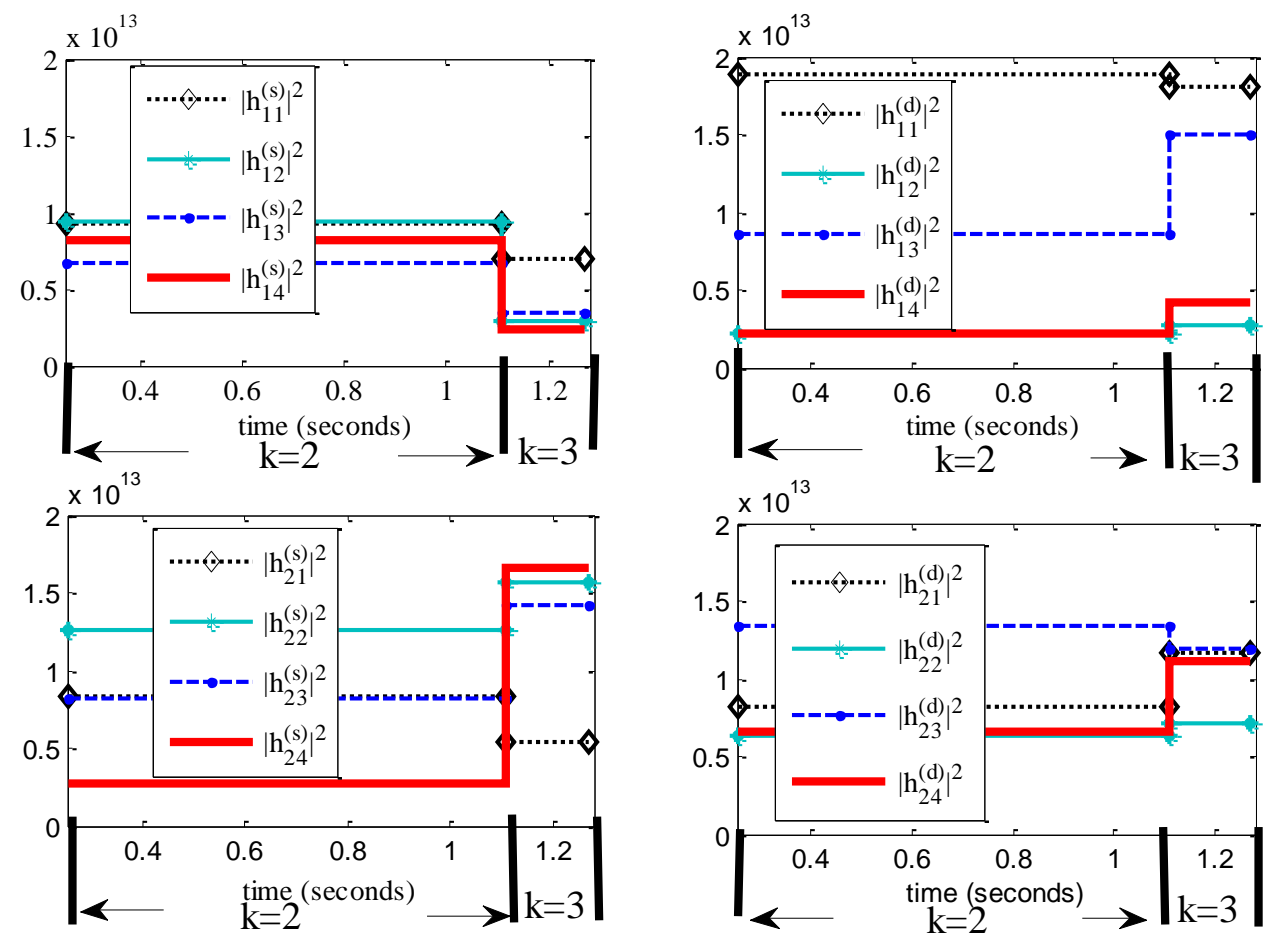

Figure 8. Channel Gains in the 2nd and 3rd Time Intervals

\subsection{Convergence Process of the Proposed Algorithm}

Thanks to the convergence properties of Lagrange dual decomposition and KuhnMunkres method, our proposed algorithm converges speedily. As a comparison, the computational complexity of the exhaustive search is $\mathrm{O}\left(\varpi^{8} \varpi^{4}\right)$, where $\varpi, \varpi^{8}$ and $\varpi^{4}$ are the iteration times of PA at one node, PA for all nodes and rate search for all S-D pairs, respectively. Obviously, the complexity of the exhaustive search will 
grow exponentially with the size of the network scale, which is unacceptable when the network is large.

\section{Conclusions}

We investigate the energy efficient energy harvesting P-TWR networks. With the fractional programming and variable relaxation, we convert the optimization into a convex one. Lagrange dual decomposition and Kuhn-Munkres algorithm is employed to obtain the optimal power allocation, relay selection and rate policies across multiple time intervals. It is shown that the harvested energy is not necessarily depleted and part of the energy is saved and consumed in later time intervals. EE comparison among different transmission strategies shows that our algorithm can provide a higher EE and has a lower computation complexity. It is also shown that a high pre-defined rate threshold can cost significant EE penalty. Since our algorithm updates the rate and power once new energy arrives or channel gain varies, it can adapt to any changes in the channels and energy arrivals and thus is considerably flexible.

\section{Appendix}

\section{A. Prove that P3 is convex}

First, the objective function is clearly convex with respect to all the variables.

For the constraints, it is also easy to find that $\mathrm{F}_{E}$ and $\mathrm{F}_{M}$ are convex sets. In what follows, we focus on proving the convexity of the region $\mathrm{F}_{R}$. Take (15) as an example. The proofs for (16) (19) follow the same approach.

It is obvious that $\log \left(1+\mid h_{i j, k}^{(s)} \& \tilde{p}_{i j, k}^{(s)} / \mathrm{D}_{1, k}\right)$ is concave. Since taking the perspective transformation for concave term will preserve the concavity, then $u_{i j, k} \log \left(1+\frac{\mid h_{i j, k}^{(s)} \ell \tilde{p}_{i j, k}^{(\mathrm{s})}}{u_{i j, k} \mathrm{D}_{1, k}}\right)$, as the perspective transformation, is also concave. Further, the sum of concave functions preserves concavity. Then the convexity of $R_{i, k}^{(\mathrm{s})}-\stackrel{\mathrm{a}}{\mathrm{a}}_{j=1}^{N} u_{i j, k} \log \left(1+\frac{\mid h_{i j, k}^{(s)} P^{2} \tilde{p}_{i j, k}^{(s)}}{u_{i j, k} \mathrm{D}_{1, k}}\right)$ is proved.

\section{B. Proof of Proposition 1}

From (27) and (28), we obtain that

$$
\tilde{p}_{i j, k}^{(s)}=\frac{u_{i j, k} a_{i j, k}^{(s)} \tilde{p}_{i j, k}^{(d)}}{b_{i j, k}^{(s)} \tilde{p}_{i j, k}^{(d)}+u_{i j, k} c_{i j, k}^{(s)}}
$$

By substituting (38) into (28), we have

$$
f_{3} *\left(\widetilde{p}_{i j, k}^{(d)}\right)^{2}+u_{i j, k} f_{2} * \tilde{p}_{i j, k}^{(d)}+\left(u_{i j, k}\right)^{2} f_{1}=0
$$

$\tilde{p}_{i j, k}^{(d *)}$ can be obtained by solving (39) according to the signs of $f_{3}, f_{2}, f_{1}$ and $f_{2}^{2}-4 f_{3} f_{1}$. Note that the convexity of P3 indicates the uniqueness of $\tilde{p}_{i j, k}^{(d *)}$. Nevertheless, it can be found that $\tilde{p}_{i j, k}^{\left(d^{*}\right)}$ is in the form of $\tilde{p}_{i j, k}^{(d *)}=u_{i j, k} p_{i, k}^{\left(d^{*}\right)}$; meanwhile, we have $\tilde{p}_{i j, k}^{(d *)}=u_{i j, k} p_{i, k}^{\left(d^{*}\right)}$ according to (23). Hence, $p_{i, k}^{(d *)}=p_{i, k}^{(d *)}$. Note $p_{i, k}^{(d *)}$ is a function of $f_{3}, f_{2}, f_{1}$ and not related with $u_{i j, k}$. This indicates that $p_{i, k}^{(d *)}$ is also independent of $u_{i j, k}$. 
In the following, we take the case when $f_{3}>0$ as an example to illustrate the calculation for $\tilde{p}_{i j, k}^{\left(d^{*}\right)}$. It can be obtained from (34) that $f_{1} £ 0$, thus we claim that $f_{2}^{2}-4 f_{3} f_{1}$ ? 0 . Further, we have

$$
\tilde{p}_{i j, k}^{(d)}=u_{i j, k} \frac{-f_{2}+\sqrt{f_{2}^{2}-4 f_{3} f_{1}}}{2 f_{3}} \geq 0
$$

and

$$
p_{i, k}^{(d *)}=p_{i, k}^{(d *)}=\frac{-f_{2}+\sqrt{f_{2}^{2}-4 f_{3} f_{1}}}{2 f_{3}} \geq 0
$$

The calculations for $\tilde{p}_{i j, k}^{\left(d^{*}\right)}$ in the other cases follow the same approach and we skip their details.

\section{Proof of Remark 2}

Assume that $R_{j}$ is matched with the $i$ th S-D pair, i.e., $u_{i j, k}=1$. Suppose another scenario, where the $S_{i}-R_{j}$ channel gain $\left|\hat{h}_{i j, k}^{(d)}\right|^{2}$ is larger than $\left|h_{i j, k}^{(d)}\right|^{2}$. Denote the optimal power at $S_{i}$ and $R_{j}$ in the assumed scenario as $\hat{p}_{i, k}^{(s)}$ and $\hat{p}_{i, k}^{(d)}$, respectively. To prove Remark 2, we only need to illustrate that $\hat{p}_{i, k}^{(d)}<p_{i, k}^{\left(d^{*}\right)}$ if $\left|\hat{h}_{i j, k}^{(d)}\right|^{2}>\left|h_{i j, k}^{(d)}\right|^{2}$. In what follows, we prove it by contradiction. That is, we assume that $\hat{p}_{i, k}^{(d)} \geq p_{i, k}^{\left(d^{*}\right)}$ if $\left|\hat{h}_{i j, k}^{(d)}\right|^{2}>\left|h_{i j, k}^{(d)}\right|^{2}$. Then based on the hypothesis, some contradictive conclusions are obtained.

when $u_{i j, k}=1$, (38) can be rewritten as

$$
p_{i, k}^{(s)}=\frac{a_{i j, k}^{(s)} p_{i, k}^{(d)}}{b_{i j, k}^{(s)} p_{i, k}^{(d)}+c_{i j, k}^{(s)}}
$$

As shown in (35), $a_{i j, k}^{(s)} \geq 0$ and $c_{i j, k}^{(s)} \geq 0$. Then if $\hat{p}_{i, k}^{(d)}>p_{i, k}^{\left(d^{*}\right)}$, we have

$$
\hat{p}_{i, k}^{(s)} \stackrel{(42)}{=} \frac{a_{i j, k}^{(s)} \hat{p}_{i, k}^{(d)}}{b_{i j, k}^{(s)} \hat{p}_{i, k}^{(d)}+c_{i j, k}^{(s)}}>\frac{a_{i j, k}^{(s)} p_{i, k}^{(d)}}{b_{i j, k}^{(s)} p_{i, k}^{(d)}+c_{i j, k}^{(s)}} \stackrel{(42)}{=} p_{i, k}^{\left(s^{*}\right)}
$$

Further, we have

$$
\frac{\delta_{i, k}^{(s)} \Delta_{1, k}}{\hat{p}_{i, k}^{(s)}}+\frac{\delta_{i, k}\left|h_{i j, k}^{(s)}\right|^{2} \Delta_{1, k}}{\left|h_{i j, k}^{(s)}\right|^{2} \hat{p}_{i, k}^{(s)}+\left|\hat{h}_{i, k}^{(d)}\right|^{2} \hat{p}_{i, k}^{(d)}}<\frac{\delta_{i, k}^{\left(s^{*}\right)} \Delta_{1, k}}{p_{i, k}^{(s)}}+\frac{\delta_{i, k}\left|h_{i j, k}^{\left(s^{*}\right)}\right|^{2} \Delta_{1, k}}{\left|h_{i j, k}^{(s)}\right|^{2} p_{i, k}^{\left(s^{*}\right)}+\left|h_{i, k}^{(d)}\right|^{2} p_{i, k}^{\left(d^{*}\right)}} \stackrel{(27)}{=} z_{i, k}^{(s)}
$$

Finally, we have

$$
\frac{\partial \mathrm{L}}{\hat{p}_{i, k}^{(s)}}=\frac{\delta_{i, k}^{(s)} \Delta_{1, k}}{\hat{p}_{i, k}^{(s)}}+\frac{\delta_{i, k}\left|h_{i j, k}^{(s)}\right|^{2} \Delta_{1, k}}{\left|h_{i j, k}^{(s)}\right|^{2} \hat{p}_{i, k}^{(s)}+\left|\hat{h}_{i, k}^{(d)}\right|^{2} \hat{p}_{i, k}^{(d)}}-z_{i, k}^{(s)}<0
$$

It can be found that $\frac{\partial \mathrm{L}}{\hat{p}_{i, k}^{(s)}} \neq 0$ from (45), which indicates that $\hat{p}_{i, k}^{(s)}$ and $\hat{p}_{i, k}^{(d)}$ cannot be the optimal power, and that the primal hypothesis is invalid. Following the same way, we can prove that $\frac{\partial p_{i, k}^{\left(s^{*}\right)}}{\partial\left|h_{i j, k}^{(s)}\right|^{2}}<0, \frac{\partial p_{j, k}^{\left(r^{*}\right)}}{\partial\left|h_{i j, k}^{(s)}\right|^{2}}<0$ and $\frac{\partial p_{j, k}^{\left(r^{*}\right)}}{\partial\left|h_{i j, k}^{(d)}\right|^{2}}<0$. 


\section{Proof of Proposition 2}

We first abstract $S_{i}-D_{i}$ pair into node $\left(S_{i}, D_{i}\right)$ and $R_{j}$ into node $\left(R_{j}\right)$. Then we have two sets $x=\left\{\left(S_{i}, D_{i}\right) \mid i=1,2, \cdots, M\right\}$ and $V=\left\{\left(R_{j}\right) \mid j=1,2, \cdots, N\right\}$, which represent the set for all S-D pair nodes and all relay nodes, respectively. If an edge connects node $\left(S_{i}, D_{i}\right)$ with node $\left(R_{j}\right)$ in the $k$ th time interval, we claim that $R_{j}$ is selected for $\left(S_{i}, D_{i}\right)$. All edges connecting a node in $X$ with another node in $v$ constitute an edge set $E_{k}$. In this way, we obtain a bipartite graph $G_{k}=\left(x ? V E_{k}\right)$. If we take $W_{i j, k}$ as the weight of an edge, then RS in the $k$ th time interval is equivalent to the MWBGM problem of $G_{k}$ due to the following facts: (i) $X$ and $v$ are two independent sets and do not have any common element; (ii) Any two edges do not share a common vertex, which is in accordance with constraint in (11). KuhnMunkres algorithm [17] can be applied to solve the optimization problem of $\max \left\{\sum_{j} \sum_{i} u_{i j, k} w_{i j, k}\right\}$.

Since $\max \sum_{k} \sum_{j} \sum_{i} u_{i j, k} w_{i j, k}=\sum_{k} \max \left\{\sum_{j} \sum_{i} u_{i j, k} w_{i j, k}\right\}$, P5 can solved by carrying out Kuhn-Munkres algorithm for each individual $G_{k}(k=1,2, \ldots K)$.

\section{Acknowledgments}

This work was supported in part by the Natural Science Basic Research Plan in Shaanxi Province of China under Grant 2016JM6062, in part by the Aerospace Science and Technology Innovation Fund of China Aerospace Science and Technology Corporation, and in part by the Shanghai Aerospace Science and Technology Innovation Fund under Grant SAST2016034.

\section{References}

[1] O. Ozel, J. Yang, and S. Ulukus, "Optimal broadcast scheduling for an energy harvesting rechargeable transmitter with a finite capacity battery," IEEE Transactions on Wireless Communications, vol. 11, no. 6, (2012), pp. 2193-2203.

[2] J. Xu and R. Zhang, "Throughput optimal policies for energy harvesting wireless transmitters with nonideal circuit power,” IEEE J. Sel. Areas Commun., vol. 32, no. 12, (2014), pp. 322-332.

[3] K. Tutuncuoglu, A. Yener, "The energy harvesting and energy cooperating two-way channel with finitesized batteries," IEEE Global Communications Conference (GLOBECOM), pp.1424-1429, (2014).

[4] K. Tutuncuoglu, B. Varan, A. Yener, "Throughput Maximization for Two-Way Relay Channels With Energy Harvesting Nodes: The Impact of Relaying Strategies," IEEE Transactions on Communications, vol. 63, no. 6, (2015), pp. 2081-2093.

[5] Nan Qi, Ming Xiao, Theodoros A. Tsiftsis, Mikael Skoglund, Phuong L. Cao, and Lixin Li. Energy Efficient Network Coding with Joint Relay Scheduling and Power Allocation [J]. IEEE transactions on Communications, vol. 64, no. 11, (2016), pp. 4506-4519.

[6] W. Chen, Z. Cao and L. Hanzo, "Maximum euclidean distance network coded modulation for asymmetric decode-and-forward two-way relaying," IET Communications, vol. 7, no. 10, (2013), pp. 988-998.

[7] D. Wang, B. Bai, W. Chen and Z. Han, "Energy efficiency maximization for secure data transmission over DF relay networks," 2015 IEEE International Conference on Communications (ICC), London, 2015, pp. 2313-2317, (2015).

[8] C. Yan, N. Yiyang and Z. Hongbo, "Performance for device-to-device communication with three-timeslot two-way amplify-and-forward relay protocol," China Communications, vol. 12, no. 11, (2015), pp. 1-11, (2015).

[9] F. Zesong, L. Chen, X. Chengwen and K. Jingming, "Joint linear processing design for distributed twoway Amplify-and-Forward MIMO relaying networks," China Communications, vol. 10, no. 7, (2013), pp. 126-133. 
[10] Z. Fei, N. Li, C. Xing and S. Gong, "Energy-efficient transceiver design for multi-pair two-way relay systems," China Communications, vol. 12, no. 9, (2015), pp. 133-140.

[11] A. Alsharoa, H. Ghazzai and M. S. Alouini, "Energy efficient design for MIMO two-way AF multiple relay networks," 2014 IEEE Wireless Communications and Networking Conference (WCNC), Istanbul, pp. 1007-1011, (2014).

[12] Yu-Chih Huang, K. R. Narayanan and Tie Liu, "Coding for Parallel Gaussian Bidirectional Relay Channels: A Deterministic Approach,” IEEE Transactions on Information Theory, vol. 62, no. 1, (2016), pp. 260-271.

[13] Yuan Liu and Xiaodong Wang, "Information and energy cooperation in OFDM relaying," 2015 IEEE International Conference on Communications (ICC), London, pp. 2506-2511, (2015).

[14] D. W. K. Ng, E. S. Lo and R. Schober, "Wireless information and power transfer: Energy efficiency optimization in OFDMA systems," IEEE Trans. Wireless Commun., vol. 12, no. 12, (2013), pp. 63526370.

[15] X. Zhou, B. Bai and W. Chen, "Greedy Relay Antenna Selection for Sum Rate Maximization in Amplify-and-Forward MIMO Two-Way Relay Channels Under a Holistic Power Model," IEEE Communications Letters, vol. 19, no. 9, (2015), pp. 1648-1651.

[16] S. Boyd and L. Vandenberghe, Convex Optimization, Cambridge University Press, (2004).

[17] S. Gao, "Graph Theory and Network Flow Theory," Higher Education Press Beijing, (2009). 